\title{
Persian Translation and Psychometric Testing of the Scales for Measuring Maternal Satisfaction in Normal and Caesarean Birth
}

\author{
Nadieh Pakari' ${ }^{1}$, Shahnaz Tork Zahrani ${ }^{2 *}$, Maliheh Nasiri ${ }^{2}$ and Zohrerh Mahmoodi ${ }^{3}$ \\ ${ }^{1}$ School of Nursing and Midwifery, Shahid Beheshti University of Medical Sciences, \\ International Branch, Tehran, Iran. \\ ${ }^{2}$ School of Nursing and Midwifery, Shahid Beheshti University of Medical Sciences, Tehran, Iran \\ ${ }^{3}$ Professor of Nursing and Midwifery faculty, Alborz University of Medical Sciences, Karaj, Iran.
}

http://dx.doi.org/10.13005/bbra/2038

(Received: 05 January 2016; accepted: 06 February 2016)

\begin{abstract}
Childbirth is a crucial experience in women's life as it has a substantial psychological, emotional and physical impact. A childbirth satisfaction is important to the mother, infant's health and well-being, and mother-infant relationship. Furthermore, it is useful for the care providers to guarantee the best preparation, health service and support to childbearing women. The present study was conducted to validate and examine the factor structure of the Persian version of the scales for measuring maternal satisfaction in normal and caesarean birth. 43-item SMMS -Normal birth and 42-item SMMSCaesarean birth were first translated by specialists into Persian. The questionnaires' validity was determined by using face, content, and criterion and construct validity. Reliability of questionnaires was examined by using Cronbach's alpha. Confirmatory factor analysis was performed in LISREL8.8 and SPSS 21.Participants included healthy Iranian postpartum women who refer to selected hospitals for delivery. Hospitals included social security and university hospitals and selected through the random cluster sampling method. The results of validity and reliability assessments of the questionnaires were acceptable. Cronbach's alpha calculated showed a high internal consistency of 0.89 for SMMS-Normal birth and $\mathbf{0 . 8 4}$ for SMMS- Caesarean birth. The confirmatory factor analysis using the c2, CMIN/DF, IFI, CFI, NFI and NNFI indexes showed the 10 factor model to be the best fitted model for explaining the data. The scales are valid and reliable tools for evaluating Iranian women's experiences in labour and the early postpartum period.
\end{abstract}

Key words: Childbirth, Women's satisfaction, Scales, Persian Translation, Validity, Factor Structure.

Childbirth as a phenomenon leading to birth of a child is an inseparable part of human life. This phenomenon has an increasing growth across the world so that nowadays childbirth is the first reason for being admitted in hospitals and health centers(Pfuntner, Wier et al. 2013). Childbearing is one of the most important events in women's lives. Not only is parturition the transition to motherhood, but childbirth itself has a substantial

\footnotetext{
* To whom all correspondence should be addressed.
}

physical and emotional impact(Kuo, Lin et al. 2010, Bertucci, Boffo et al. 2012). Therefore, the examination of maternal care quality during childbirth is of a high significance (Salmon, Miller et al. 1990, Dencker, Taft et al. 2010, Jafari and Khalifegari 2010).

Since 1990, patients' satisfaction has been considered as an important quality criterion in American health and treatment centers(Mitchell, Ferketich et al. 1998). Since 2002, in Iran also, health and treatment ministry aiming at doing its main mission has obliged all hospitals to make periodical assessment of patients' satisfaction and to take 
necessary measures for increasing clients' satisfaction(Jafari and Khalifegari 2010). In recent years, the analysis of perceived satisfaction by users of health-care systems has become more important (Sadjadian, Kaviani et al. 2002, Amerion, Ebrahimnia et al. 2009). assessments of women's satisfaction with their care during labour and childbirth are relevant to health-care providers, administrators and policy makers to assess the quality of care, make decisions about the organisation and provision of health-care services, avoid malpractice litigation and maintain a competitive edge in the health-care arena (Goodman, Mackey et al. 2004, Gungor and Beji 2012).

Measuring satisfaction is not a simple task, it is a multidimensional concept which is affected by affective responses triggered by specific experiences, and by the cognitive assessments that individuals make of it. However ,we can generally state that the evaluation of satisfaction by questionnaire is an effective system that also enables objective comparison among women and institutions , and even detailed analysis of the various aspects involved(Christiaens and Bracke 2007, Fair and Morrison 2012). Various instruments have been developed to assess women's satisfaction with labour and childbirth. These have all been designed and standardised for groups of English, Spanish, Turkish women. Childbirth perception questionnaire (Padawer, Fagan et al. 1988), birth experience questionnaire (Salmon, Miller et al. 1990), child birth experience questionnaire (Dencker, Taft et al. 2010), women's views of birth labor satisfaction questionnaire (Smith 2001), women's evaluations of their labor and delivery nurses (Goodman, Mackey et al. 2004), scales for measuring maternal satisfaction in normal and caesarean birth are introduced in various studies for evaluation of maternal satisfaction of childbirth. Among these, this is Mac kay's childbirth scale of satisfaction that is applied in Iran (Dolatian, Sayyahi et al. 2008, Jafari and Khalifegari 2010). Most of the above mentioned questionnaires are designed and presented for women's childbirth perception and satisfaction evaluation (Smith 2001); these are specifically standardized and psychometrically designed for English, Spanish, and Italian population (Mitchell, Ferketich et al. 1998, Smith 2001, Bertucci, Boffo et al. 2012, Gungor and Beji 2012).

Since instruments applied in a study need to have enough and robust psychometric characteristics and they also need to be able to scale various aspects of the topic, this study is aimed on psychometrical characteristic examination of multidimensional evaluation questionnaire related to maternal satisfaction of normal and caesarian childbirth by Miss Gungor. This study is conducted in Turkey for three reasons; first, this is a validated instrument designed for examining various aspects of psychometry; second, this instrument conducts a multidimensional examination of normal and caesarean childbirth separately (these characteristics are absent in Mc Cay's instrument); third, childbirth culture of Turkey is closer to that of Iran in comparison to European and American countries.

\section{MATERIALSANDMETHODS}

The present psychometric methodological research was conducted in 2015.

\section{Participants}

The samples were selected from postpartum care units of university and social security hospitals in Tehran and Bushehr, through the stratified cluster sampling method in Tehran Therefore, Tehran was divided into 5 regions of north, south, east, west . A hospital was randomly selected from each region, and 3 university and social security hospitals in bushehr. The sample included 430 (215 normal, 215 caesarean) low-risk postpartum women who gave birth to a single healthy fetus over 37 weeks of gestation during the study period, having a singleton embryo and a normal course of pregnancy (Without every complication that is lead to hospitalization) and who agreed to participate in the study.

\section{Stage 1-Translation of Questionnaire}

The researcher translated the questionnaire into Persian through the 3-step method after obtaining the designer's(Acquadro, Lafortune et al. 2003). To carry out the 3 steps, the questionnaire was translated into Persian separately by two; then, Persian questionnaire was translated into English again by another English and Persian language expert; eventually, a committee of team members prepared the final 
Persian version of the questionnaire according to the rules of semantic, terminological, experiential and comprehensive parity.

Scales for Measuring Maternal Satisfaction in Normal birth consists of 10 subcategories: Perception of Health Professionals (4 items), Nursing Care in Labor (2items), Comforting(4 items), Information and Involvement in Decision Making (8 items), Meeting Baby(3 items), Postpartum Care(6 items), Hospital Room(4 items), Hospital Facilities(3 items), Respect for Privacy (4items), Meeting Expectations(5items), and Scales for Measuring Maternal Satisfaction in ceaseran birth consists of 10 subcategories: Perception of Health Professionals (5 items), Nursing Care in Labor(2items), Comforting(3 items), Information and Involvement in Decision Making (8 items), Meeting Baby(3 items), Postpartum Care(6 items), Hospital Room(3 items), Hospital Facilities(3 items), Respect for Privacy(4items). Meeting Expectations(5items)(Gungor and Beji 2012).

\section{Stage 2- Psychometric Analysis of Instruments}

This step was dedicated to evaluation of the psychometric properties of the questionnaire. The present study examined the validity and reliability of Scales for Measuring Maternal Satisfaction in Normal and Caesarean Birth (SMMS-normal birth and SMMS- caesarean birth)(Schneider and Whitehead 2013). For determining the questionnaire's validity, the face, content, criterion and construct validity were examined and the internal consistency and stability were examined to determine its reliability.

\section{Face Validity}

Face validity has both qualitative and quantitative aspects. In this study face validity was examined through both qualitative and quantitative approach (Wright \& Stone, 1999). Face validity emphasizes the viewpoints of both

the target group(Polit and Beck 2013) and the experts and the present study also used the viewpoints of both the target group and the experts (Hajizadeh and Asghari 2011, Shultz, Whitney et al. 2013) To examine the face validity through the qualitative approach, used the viewpoints of the expert group consisting of 12 members ( 2 psycometrist, 3 reproductive health doctors, 3 professors of midwifery, 2 nurse with a $\mathrm{PhD}, 2$ professors of nursing and midwifery management) and also of 30 postpartum women (Wilson, 1985) admitted to a selected hospital in Tehran and bushehr were randomly selected and interviewed face to face about the questionnaire items. This part examined the levels of difficulty, irrelevance and ambiguity.

To eliminate inappropriate items in the next step, the importance of each item was determined using the quantitative item score impact method. This step used the viewpoints of the target group that had participated in the qualitative step . The item score impact was calculated using the following equation: Item Score Impact= Frequency $(\%) \times$ Importance. Each item was scored within the 5-point Likert scale (Not important at all $=1$ to absolutely important $=5$ ) by participants (30 postpartum women). The score impact was calculated for each item. Items with a score of 1.5 or higher were retained and deemed appropriate to enter the next step of analysis(Somekh and Lewin 2005, Drost 2011, Hajizadeh and Asghari 2011).

\section{Content Validity}

Content validity was also examined through both qualitative and quantitative approach. The present study determined the qualitative content validity using the experts' opinions(Wilson 1985, Drost 2011). The experts first performed a qualitative examination of the questionnaire based on the rules of grammar, wording, item allocation and proper scaling and then presented their feedback.

For the quantitative examination of the content validity, two indexes Content Validity Ratio (CVR) and Content Validity Index (CVI) were used. To calculate the CVR, the 12 experts were requested to score each of the 43 items of the questionnaire SMMS-normal birth and each of the 42 items of the questionnaire SMMS- caesarean birth on the basis of a 3-point Likert scale (It is necessary=1, It is useful but not necessary=2, It is not necessary=3). If the calculated CVR was larger than its corresponding value in Lawshe's table (based on the assessments of the 12 experts), i.e. larger than 0.56, that item was retained in the questionnaire with the statistical significance level of $\mathrm{P}<0.05$ (Lawshe, 1975). Then, CVI was determined base on Waltz and Bausell content validity index(Waltz and Bausell 1981). The same group of experts expressed their opinions about the relevance, simplicity and comprehensibility of 
each item of questionnaire in a 4-point Likert scale. If an item's calculated CVI was larger than 0.79, it was deemed appropriate; if, however, it was between 0.7 and 0.79 , the item was questionable and required revision; and if the CVI was lower than 0.7 , the item had to be removed entirely (Kellar and Kelvin 2012, Polit and Beck 2013, Schneider and Whitehead 2013). If the average CVI for the entire questionnaire was equal to or higher than $90 \%$, then the S-CVI/Ave and consequently the scale were acceptable(Hajizadeh and Asghari 2011).

\section{Criterion Validity}

In terms of scale development and validation, evidence of convergent validity is typically provided from correlations between the new measure being testing and an existing measure(Netemeyer, Bearden et al. 2003, Öner 2006). With this aim, the Mackey Childbirth Satisfaction Rating Scale was used in this study to establish the expected positive correlation (convergent validity) between women's satisfaction with nursing/midwifery care and overall satisfaction with care during their hospital stay. The 2 scales (SMMS-normal birth / SMMScaesarean birth and MCSRS) were used to measure 220 randomly-selected postpartum women (110 normal, 110 caesarean) and were then compared, indicating a favorable correlation, which also confirmed the predictive validity of the questionnaire.

\section{Construct Validity}

Factor analysis is a useful analytical tool that can identify potential underlying dimensions/ subscales in a scale(Munro 2005), for constract validity, because specifying items and subscales, factor analysis was used(Kahn 2006). The data were examined for the confirmatory factor analysis in SPSS-21 and AMOS. Given the AMOS output consist of Chi-square (?2)test, Chi-square Degree of Freedom Ratio ( Normalized chi-square CMIN/ DF), Comparative Fit Index (CFI), Incremental Fit Index (IFI), Tucker-Lewis Index or the Non Normalized Fit Index (NNFI), Bentler-Bonett Index or Normalized Fit Index (NFI), and Root Mean Square Error of Approximation (RMSEA) were used for Confirmatory Factor Analysis(Strauss and Smith 2009, Ghasemi 2010).

\section{Questionnaire's Reliability}

The questionnaire's reliability was determined through the internal consistency and the stability measures. Cronbach's alpha is a famous method for measuring internal consistency(LoBiondo-Wood, Haber et al. 2013, Polit and Beck 2013). ) In this respect, 80 postpartum women (40normal. 40 ceaserean) randomly selected from the selected hospitals and the questionnaires were filled out by them. The internal consistency of questionnaire (Cronbach's alpha) was determined. The internal consistency was acceptable if the Cronbach's alpha measurement was equal to or greater than 0.70 . For assessing of stability of SMMS-normal birth / SMMS- caesarean birth, Test re Test method was examined. In this respect 80 postpartum women ( 40 normal, 40 ceaserean) randomly selected from two of the selected hospitals, who then filled out the questionnaire over 2 stages with a 14-day interval(Hajizadeh and Asghari 2011). The obtained scores were then compared with each other using Pearson's correlation coefficient. An optimum correlation coefficient is higher than 0.70 (Drost 2011, Kellar and Kelvin 2012).

In order to respect the ethical considerations, the study was conducted upon receiving the consent of the presidents of Shahid Beheshti University, University of bushehr, Social Security Organization and the hospital directors. Participants were given all the necessary information prior to participation in the study and were also ensured of the confidentiality of their information and they could withdraw from the study whenever they liked. Then, participants gave their informed verbal consent to the researcher before they participated in the study.

\section{Findings}

The mean (standard deviation) ages of women were 28 (5.2) and 29 (5.1) years in the normal and caesarean birth groups, respectively. Demographic and obstetric characteristics are presented in table 1.

\section{Findings of content validity}

Assessment of the qualitative content validity confirmed the items in accordance with the expert viewpoints. In the assessment of the quantitative content validity, item CVR scores ranged from 0.38 to 1 in form of the SMMS-normal birth, whereas item CVR scores ranged between 0.69 and 1 in the draft form of the SMMS-caesarean birth. Items with alow CVR score(o0.56) were removed from the tools by deleting one items from 
Table 1. Demographic and obstetric characteristics of women

\begin{tabular}{|c|c|c|c|c|c|}
\hline & & \multicolumn{2}{|c|}{ Normal birth } & \multicolumn{2}{|c|}{ Ceasarean birth } \\
\hline & & $\mathrm{n}$ & $\%$ & $\mathrm{n}$ & $\%$ \\
\hline & Primary school & 15 & 6.9 & 12 & 5.6 \\
\hline & guidance school & 24 & 11.1 & 20 & 9.3 \\
\hline \multirow[t]{3}{*}{ Level of education } & High school & 76 & 35.2 & 70 & 32.6 \\
\hline & University & 100 & 46.3 & 111 & 51.6 \\
\hline & housewife & 132 & 61.1 & 116 & 54 \\
\hline \multirow[t]{4}{*}{ Occupational status } & worker & 7 & 3.2 & 10 & 4.7 \\
\hline & employee & 50 & 23.1 & 63 & 29.3 \\
\hline & Others & 26 & 12.1 & 24 & 11.1 \\
\hline & 1 & 79 & 36.6 & 92 & 42.8 \\
\hline \multirow[t]{4}{*}{ Number of pregnancy } & 2 & 82 & 38 & 76 & 35.3 \\
\hline & 3 & 37 & 17.1 & 35 & 16.3 \\
\hline & 4 & 14 & 6.5 & 11 & 5.1 \\
\hline & 5 & 3 & 1.4 & 1 & 0.5 \\
\hline \multirow[t]{3}{*}{ Economic status } & Low income & 22 & 10.2 & 15 & 7 \\
\hline & Middle income & 151 & 69.9 & 125 & 58.1 \\
\hline & High income & 42 & 19.4 & 75 & 34.8 \\
\hline
\end{tabular}

the SMMS-normal birth and no item was therefore removed from the SMMS- caesarean birth.

\section{Findings of Face Validity}

Assessment of the qualitative face validity confirmed all the 42 items from two tools. In the assessment of the quantitative face validity, the impact item score for both the target and the expert groups was higher than 1.5 and no single item was therefore eliminated.

\section{Findings of Criterion Validity}

Scales established their convergent validity with significant correlations with the MCSRS (SMMS-normal birth: $r=0.724$, $p=0.000$; SMMS-caesarean birth: $r=0.686, p=0.000$ ).

\section{Findings of Construct Validity}

The results of the confirmatory factor analysis confirmed the model and the questionnaire items showed favorable fit indexes For the SMMSnormal birth, the results of the fit indices were $\mathrm{x}^{2} /$ $\mathrm{df}=2 / 09$, root mean square error of approximation $($ RMSEA $)=0.070$, comparative fit index $(\mathrm{CFI})=0.97$, non-normed fit index(NNFI) $=0.96$, goodness of fit index $(\mathrm{GFI})=0.84$. Similarly, for the SMMScaesarean birth, the results of the fit indices were $\mathrm{x}^{2} / \mathrm{df}=2 / 082 / 08,(\mathrm{p}=0.000), \mathrm{RMSEA}=0.069, \mathrm{CFI}=0.97$, $\mathrm{NNFI}=0.95, \mathrm{GFI}=0.84$. Assessment of acceptable model fit in the confirmatory factor analysis concluded that the model given had the best fit according to the results of $\mathrm{x}^{2} / \mathrm{df}$, RMSEA, CFI

\section{,NNFI and GFI.}

\section{Reliability}

The internal consistency and stability of the questionnaire determined the reliability. Both scales had good internal reliability with Cronbach's alpha coefficients of 0.91. Cronbach's alpha coefficients for the subscales ranged from 0.71 to 0.89 for the SMMS-normal birth and from 0.70 to 0.82 for the SMMS-caesarean birth. The stability of the questionnaires was examined through the test-retest method and was deemed favorable ( $r=0.99$ and $\mathrm{p}=0.000$ for SMMS- normal birth, $\mathrm{r}=0.98$ and $\mathrm{p}=0.000$ for SMMS- Caesarean birth).

\section{DISCUSSION}

The multidimensional structure of factors that affect satisfaction has been emphasised in several studies that assessed women's birth experiences and satisfaction with care(Janssen, Dennis et al. 2006, Waldenström, Rudman et al. 2006). The instruments include different dimensions depending on the authors' theoretical concept of 'satisfaction'. One such instrument, the Childbirth Experience Questionnaire includes four factors: own capacity, professional support perceived safety, and participation (Dencker, Taft et al. 2010). The Birth Experience Questionnaire comprises three subscales (fulfillment, distress and 
difficulty)(Salmon, Miller et al. 1990). and the Childbirth Experience and Satisfaction Questionnaire contains eight subscales (conditions and care, positive experience, negative experience, relaxation, social support, positive partner support, worries and post partum)(Costa, Figueiredo et al. 2004). The sheer variety of instruments and components used to assess the 'satisfaction' construct make it difficult to find a common theoretical factorial structure that determines satisfaction with childbirth (Bertucci, Boffo et al. 2012).

Women' Satisfaction or Dissatisfaction of their birth experiences are associated with many personal and institutional factors, and the support they received during birth. Maternal satisfaction and related factors are often focused on issues including sociodemographic obstetric factors, organisation of maternity services, expectations and birth plan, antenatal preparation, communication with health professionals, privacy, medical procedures, midwifery/nursing care, emotional support, pain relief, presence of significant others during birth, being informed, participation in decision making, infant care, postpartum care and continuity of care(Janssen, Dennis et al. 2006, Christiaens and Bracke 2007, Rudman, El Khouri et al. 2007).

The model proposed by Miss Ganger et al. is an applicable and comprehensive model(Gungor and Beji 2012). In this scale, "woman's perception of health professionals" has been considered as a separate area and measure, because most women are worry about their relationship with medical staff during childbirth. "meeting baby" are also confirms that the link between mother and child has been considered firmly; being in harmony with Iranian culture. This area includes the first breastfeeding; the importance of its colostrums is being mentioned in various studies(Proctor 1998, Gungor and Beji 2012). "Information and Involvement in Decision Making" is a measure that confirms value of receiving information and making informed choice for women. Also, this area is a concept in which mother has the feeling of controlling and understanding her situation(Rudman, El Khouri et al. 2007, Oshima Lee and Emanuel 2013). During the postpartum period, the protection and assistance of family and medical staff is an important issue impacting on women' satisfaction of received care. Then, considering "postpartum care" area shows that women make a difference between care received before and after childbirth. Women' needs are fully different during these two periods(Hildingsson and Thomas 2007, Choi 2012). Being respectful of individual's privacy as a separate factor has been considered. Janssen et al. (2006) in a study emphasis that respecting women' privacy is effective on women' satisfaction of childbirth, because women try to protect their privacy and they don't want it to be disturbed by organizations, therapists, or strangers (Ahmed and Yasin, Janssen, Dennis et al. 2006, Hollins Martin and Fleming 2011). "meeting expectations" area is a strong predictor criterion of woman's satisfaction from her childbirth. The measure related to satisfaction of physical environment has been divided into hospital room and general facilities of the hospital. This are mentions the importance of individual's comfort in childbirth room and general units of the hospital; focusing on the importance of special services meeting women' needs in the hospital(Hollins Martin and Fleming 2011).

As indicated in the results, there is a meaningful relationship between the questionnaires factors and the instrument in general which shows that women' satisfaction of normal and caesarian childbirth is fully related to women' perception of Health Professional, midwifery care, readiness for caesarian, meeting baby, respect for privacy, meeting the expectations, hospital facilities, hospital room, information and participation in making decision, and peace and comfort. Gunger et al. also in her regression model showed that some social, managerial, and supportive factors are good determinants for women' satisfaction assessment of childbirth(Gungor and Beji 2012).

\section{CONCLUSION}

In general, we can conclude from the results of present study that the final draft of "questionnaire of measuring maternal satisfaction in normal and caesarean birth" is acceptable from psychometry point of view. This instrument can be applied in assessing women's satisfaction of normal and caesarian childbirth aiming at improving hospitals' and health centers' services. The 
application of this scale is simply feasible and it can be completed in a short time by different individuals with different knowledge levels and in different ages with different pregnancy times. It is worth mentioning that each of the measures of the present scale can be used as a separate scale in examining a special dimension of women' satisfaction in different studies. Comparing the results of the present study to those of other studies on women' satisfaction of childbirth assessment in Iran shows that there is no difference between Iranian culture and Turkish culture; and also in this scale the clause of "presence of husband beside pregnant woman" is absent and this fact is consistent with Iranian culture. The other importance of this scale is differentiating normal and caesarian childbirth and this fact that testing and assessing them has been conducted by means of recommended scaling measures for psychometry characteristics. The size of the sample was sufficient. The Homogenous distribution of the characteristics of Iranian women' demography is considered. This study is conducted in social security and university hospitals in Tehran and Bushehr which is a sufficient reason for robustness of psychometric of the scales.

\section{ACKNOWLEDGEMENTS}

The present study was part of a Master's thesis with an Ethics Committee license Code Ir.sbmu.rec.1394.122, written at the International Branch of Shahid Beheshti University. Hereby, we would like to express our gratitude to Dr. Ilkay Gungor assistant professor of Department of Obstetrics and Gynaecologic Nursing School of Istanbul University, for giving permission to translate the SMMS-Normal and Caeserean Birth.

\section{REFERENCES}

1. Acquadro, C., et al.. "Quality of life in multiple sclerosis: translation in French Canadian of the MSQoL-54." Health and quality of life outcomes 2003; 1(1): 70.

2. Ahmed, H. M. and M. Y. Yasin “Mother's satisfaction regarding nursing care during labor, delivery and postpartum in Maternity Teaching Hospital in Erbil city.” Impact of a health educational program on knowledge of female nursing staff and students regarding breast
Cancer prevention and early detection in Erbil city: 16

3. Amerion, A., et al. "Inpatient and outpatient satisfaction of a military hospital.” Journal Mil Med 2009; 11(1): 37-44.

4. Bertucci, V., et al., "Assessing the perception of the childbirth experience in Italian women: a contribution to the adaptation of the Childbirth Perception Questionnaire.” Midwifery 2012; 28(2): 265-274.

5. Choi, J., "Satisfaction and demand of postpartum care program among the user in Sanhujoriwon (postpartum care center)[master's thesis].” Pocheon: CHA University, 2012.

6. Christiaens, W. and P. Bracke, "Assessment of social psychological determinants of satisfaction with childbirth in a cross-national perspective." BMC pregnancy and childbirth 2007; 7(1): 26.

7. Costa, R., et al., "Childbirth Experience and Satisfaction Questionnaire (CESQ).” Psicologia: Saú de \& Doenc-as 2004; 5: 159-187.

8. Dencker, A., et al., "Childbirth experience questionnaire (CEQ): development and evaluation of a multidimensional instrument." BMC pregnancy and childbirth 2010; 10(1): 81.

9. Dolatian, M., et al., "Satisfaction Rate of Normal Vaginal Delivery and Its Relative Factors among Childbearing Women in "Mahdiye, Tehran" and "Shaheed Chamran, Boroujerd" Hospitals, 1385.” Pajoohandeh Journal 2008; 13(3): 259268.

10. Drost, E. A., "Validity and reliability in social science research." Education Research and Perspectives 2011; 38(1): 105.

11. Fair, C. D. and T. E. Morrison., "The relationship between prenatal control, expectations, experienced control, and birth satisfaction among primiparous women.” Midwifery 2012; 28(1): 39-44.

12. Ghasemi, V., "Structural equation modeling in social researches using Amos Graphics.” Tehran: JameeShenasan Publications (in Persian), 2010.

13. Goodman, P., et al., "Factors related to childbirth satisfaction.” Journal of advanced nursing 2004; 46(2): 212-219.

14. Gungor, I. and N. K. Beji., "Development and psychometric testing of the scales for measuring maternal satisfaction in normal and caesarean birth.” Midwifery 2012; 28(3): 348-357.

15. Hajizadeh, E. and M. Asghari., "Statistical methods and analyses in health and biosciences a research methodological approach.” Tehran: Jahade Daneshgahi Publications, 2011.

16. Hildingsson, I. and J. E. Thomas, "Women's perspectives on maternity services in Sweden: processes, problems, and solutions.” Journal of 
Midwifery \& Women's Health 2007; 52(2): 126133.

17. Hollins Martin, C. and V. Fleming., "The birth satisfaction scale.” International journal of health care quality assurance 2011; 24(2): 124-135.

18. Jafari, G. and S. Khalifegari., "Danaii k, Dolatshahi P, Ramezani M, Roohparvar R, Sabaghiyan PA." Hospital accreditation standards in Iran. Tehran: Sound Publication Center, 2010.

19. Janssen, P. A., et al., "Development and psychometric testing of the care in obstetrics: measure for testing satisfaction (COMFORTS) scale.” Research in nursing \& health 2006; 29(1): 51-60.

20. Kahn, J. H., "Factor analysis in counseling psychology research, training, and practice principles, advances, and applications.” The Counseling Psychologist 2006; 34(5): 684-718.

21. Kellar, S. P. and E. A. Kelvin, Munro's statistical methods for health care research, Wolters Kluwer Health/Lippincott Williams \& Wilkins, 2012.

22. Kuo, S.-C., et al., "Evaluation of the effects of a birth plan on Taiwanese women's childbirth experiences, control and expectations fulfilment: a randomised controlled trial." International journal of nursing studies 2010; 47(7): 806-814.

23. LoBiondo-Wood, G., et al., Study Guide for Nursing Research: Methods and Critical Appraisal for Evidence-Based Practice, Elsevier Health Sciences 2013.

24. Mitchell, P. H., et al., "Quality health outcomes model.” Image Indianapolis 1998; 30: 43-46.

25. Munro, B. H., Statistical methods for health care research, Lippincott Williams \& Wilkins, 2005.

26. Netemeyer, R. G., et al., Scaling procedures: Issues and applications, Sage, 2003.

27. Öner, N., Türkiye’de kullanýlan psikolojik testlerden örnekler (Samples of Psychological Tests used in Türkiye, Ýstanbul: Boðaziçi University Press, 2006.

28. Oshima Lee, E. and E. J. Emanuel., "Shared decision making to improve care and reduce costs.” New England Journal of Medicine 2013l 368(1): 6-8.

29. Padawer, J. A., et al., "Women’s psychological adjustment following emergency cesarean versus vaginal delivery." Psychology of Women Quarterly 1988; 12(1): 25-34.

30. Pfuntner, A., et al., "Most frequent conditions in US hospitals, 2011; 2013.”

31. Polit, D. F. and C. T. Beck. Essentials of nursing research: Appraising evidence for nursing practice, Lippincott Williams \& Wilkins, 2013.

32. Proctor, S., "What determines quality in maternity care? Comparing the perceptions of childbearing women and midwives.” Birth 1998; 25(2): 85-93.

33. Rudman, A., et al., "Women's satisfaction with intrapartum care-a pattern approach.” Journal of advanced nursing 2007; 59(5): 474-487.

34. Sadjadian, A., et al., "Satisfaction with breast clinical care, 2002"

35. Salmon, P., et al., "Women's anticipation and experience of childbirth: the independence of fulfilment, unpleasantness and pain.” British Journal of Medical Psychology 1990; 63(3): 255259.

36. Schneider, Z. and D. Whitehead, Nursing and midwifery research: Methods and appraisal for evidence-based practice, Elsevier Australia, 2013.

37. Shultz, K. S., et al., Measurement theory in action: Case studies and exercises, Routledge, 2013.

38. Smith, L. "Development of a multidimensional labour satisfaction questionnaire: dimensions, validity, and internal reliability." Quality in Health Care 2001; 10(1): 17-22.

39. Somekh, B. and C. Lewin. Research methods in the social sciences, Sage, 2005.

40. Strauss, M. E. and G. T. Smith, "Construct validity: Advances in theory and methodology.” Annual review of clinical psychology 2009; 5: 1.

41. Waldenström, U., et al.. "Intrapartum and postpartum care in Sweden: women's opinions and risk factors for not being satisfied.” Acta obstetricia et gynecologica Scandinavica 2006; 85(5): 551-560.

42. Waltz, C. F. and B. R. Bausell., Nursing research: design statistics and computer analysis, Davis FA, 1981.

43. Wilson, H. S., Research in nursing, AddisonWesley Publishing Company Medical/Nursing Division, 1985. 